\title{
Especialización, concentración y aglomeración espacial de los servicios intensivos en conocimiento en España*
}

\author{
Severino Escolano-Utrilla \\ Ana Isabel Escalona-Orcao \\ Universidad de Zaragoza. Departamento de Geografía y Ordenación del Territorio. \\ Grupo de investigación GEOT (IUCA) / Grupo de investigación GEDETUZ (IUCA) \\ severino@unizar.es \\ aescalon@unizar.es
}

Recibido: mayo de 2015

Aceptado: marzo de 2016

\section{Resumen}

El uso intensivo de conocimiento, de creatividad y de información caracteriza a un conjunto heterogéneo de actividades de servicio (acrónimo en inglés: KIS o KISA) muy ramificadas por el tejido social y productivo. La localización es un factor crucial para el desenvolvimiento de los KIS, que tienden, en general, a aprovechar las oportunidades de las economías de urbanización. Con estos supuestos, en el presente trabajo se estudia la localización de los KIS en España a varias escalas mediante indicadores de autocorrelación espacial (LISA). Los resultados demuestran que los KIS presentan un grado de concentración mayor que el de la población, y más acusado que en otros países y en otras actividades de la economía del conocimiento. Los KIS se aglomeran en grandes áreas metropolitanas, con variaciones que dependen de la intensidad de conocimiento invertido en la producción de los servicios.

Palabras clave: servicios intensivos en conocimiento; KIS; aglomeraciones urbanas; LISA; España

Resum. Especialització, concentració i aglomeració espacial dels serveis intensius en coneixement a Espanya

L'ús intensiu de coneixement, de creativitat i d'informació caracteritza un conjunt heterogeni d'activitats de serveis (acrònim en anglès: KIS o KISA) molt ramificades pel teixit productiu i social. La localització és un factor clau per al desenvolupament dels KIS, que tendeixen, en general, a aprofitar les oportunitats de les economies d'urbanització. Amb aquests supòsits, en el present treball s'estudia la localització dels KIS a Espanya a diverses escales mitjançant indicadors d'autocorrelació espacial (LISA). Els resultats demostren que els KIS presenten un grau de concentració més elevat que el de la població, i que és més acusat que en altres països i en altres activitats de l'economia del coneixement. Els KIS s'aglomeren en grans àrees metropolitanes, amb variacions que depenen de la intensitat de coneixement invertit en la producció dels serveis.

Paraules clau: serveis intensius en coneixement; KIS; aglomeracions urbanes; LISA; Espanya

* Proyecto de investigación Los clústeres de actividades creativas en las áreas semiurbanas y rurales españolas, seleccionado en la convocatoria 2012 del Plan Nacional de I+D+I, Ministerio de Economía y Competitividad (código: CSO2012-31650). 
Résumé. Spécialisation, concentration et agglomération spatiale des services à forte intensité de connaissances en Espagne

L'utilisation intensive de la connaissance, de la créativité et de l'information caractérise un ensemble hétérogène de services à forte intensité de connaissances (SFIC, angl. KIS ou KISA) très étendues dans le tissu social et productif. La localisation est l'une des clés du développement des SFIC/KIS qui visent, en général, à l'exploitation des opportunités des économies d'urbanisation. Partant de ces hypothèses, notre travail examine la localisation des SFIC/KIS en Espagne, à différents niveaux et en utilisant des indicateurs d'autocorrélation spatiale (LISA). Les résultats montrent que les SFIC/KIS ont un degré de concentration supérieur à celui de la population, et plus fort que celui d'autres pays ou d'autres activités de l'économie de la connaissance. Les SFIC/KIS se rassemblent dans les grandes régions métropolitaines, avec des variations en fonction de l'intensité de savoirs investis dans la production des services.

Mots-clés: services à forte intensité de connaissances; KIS; agglomérations urbaines; LISA; Espagne

\section{Abstract. Specialization, concentration and spatial agglomeration of knowledge-intensive services in Spain}

The intensive use of knowledge, creativity and information characterizes a heterogeneous set of service industries which are highly integrated with other productive activities. The location of these knowledge-intensive services (KIS), a crucial factor of their development, has a tendency to take advantage of the economies of urbanization. From these assumptions, this paper studies the location patterns of KIS in Spain at various scales using indicators of spatial autocorrelation (LISA). KIS are found to cluster in large metropolitan areas, with variations depending on the intensity of the knowledge invested in the production of services. The results show that KIS have a higher degree of concentration than the population distribution and than other activities of the knowledge economy. This trend is clearer in Spain than in other countries.

Keywords: knowledge intensive services; KIS; urban agglomerations; LISA; Spain

\section{Sumario}

\section{Introducción}

2. La intensidad de conocimiento como factor de la especialización, la concentración y la aglomeración espacial de los servicios

3. Metodología y datos
4. Los contrastes espaciales de los KIS en España: alcance de la escala y de la intensidad de conocimiento

5. Consideraciones finales

Referencias bibliográficas 


\section{Introducción}

Este artículo trata sobre la localización de los servicios intensivos en conocimiento, denominados a menudo por su acrónimo en inglés, KIS (Knowledge Intensive Services) o también KISA (Knowledge Intensive Service Activities). El estudio científico de los KIS es muy relevante debido al peso económico de estas actividades y, especialmente, a su función decisiva en el desarrollo de innovaciones y en la mejora de la competitividad y de la eficiencia de las empresas de casi todos los sectores. Además, impulsan la modernización de la sociedad en general. Su carácter estratégico se acrecienta en países como España, donde los servicios tienen un gran peso socioeconómico y más aún en periodos de crisis como el presente, ya que la posición de las economías nacionales, regionales y locales en las divisiones permanentes del trabajo a todas las escalas dependerá de la intensidad y la función de los procesos de innovación de los nuevos modelos productivos. En última instancia, las condiciones de vida de los ciudadanos se relacionan fuertemente con dichas actividades, incluso tanto como con las industriales (European Commisison, 2007: 9).

Los estudios empíricos disponibles dejan patente que la localización de estos servicios varía de forma sistemática y no lineal con el tamaño demográfico de los lugares, de modo que los aumentos de población se corresponden con unos aumentos más que proporcionales de la presencia de dichos servicios. Esta codependencia permite argumentar que los KIS se vinculan estrechamente con las externalidades producidas por las aglomeraciones urbanas, lo que da lugar a estructuras espaciales polarizadas, en las que los KIS se concentran en grandes áreas urbanas, mientras que, en las áreas rurales, predominan los servicios convencionales, sin uso intensivo de conocimiento. A su vez, los estudios realizados en las grandes aglomeraciones a mayor resolución revelan la complejidad de las configuraciones espaciales, sugiriéndose la existencia a dicha escala de procesos simultáneos de concentración y dispersión.

En la Unión Europea, es bien conocido el peso económico de los servicios y también el de los KIS. En España, se han realizado estudios sobre los KIS en determinadas autonomías (NUTS-2), pero no se han llevado a cabo trabajos específicos a escala nacional, por lo que sus patrones de localización no se conocen plenamente. Por esta y otras razones, es muy oportuno elaborar un estudio de la localización de los KIS en España. Los resultados serán valiosos para la formación de planes y políticas económicas, ya que determinados tipos de KIS forman parte, con otras actividades de producción del conocimiento, de la planificación estratégica a todas las escalas.

En este contexto, la presente investigación tiene dos objetivos específicos: 1) identificar y caracterizar a los patrones espaciales de los KIS a varias escalas, en términos de especialización, concentración y aglomeración, y 2) modelar la relación funcional entre las aglomeraciones urbanas y los KIS. En relación con los trabajos publicados sobre el tema, esta investigación presenta algunas novedades: 
a) El estudio comprende todo el territorio nacional.

b) Los análisis se han realizado con dos niveles de resolución (áreas funcionales y municipios).

c) Se ha utilizado la clasificación más reciente de los KIS, que todavía no se ha empleado en los trabajos empíricos publicados hasta hoy.

Los contenidos de este artículo están organizados en cuatro partes, además de la introducción. En la primera, se presentan los conceptos que fundamentan el enfoque y los métodos seguidos en este trabajo. En la siguiente, se describe la metodología utilizada y se caracterizan los datos. En la tercera, se exponen y se valoran los resultados de los análisis. Finalmente, a modo de conclusiones, se realizan varias consideraciones sobre los resultados.

\section{La intensidad de conocimiento como factor de la especialización, la concentración y la aglomeración espacial de los servicios}

La intensidad de conocimiento es la dimensión que articula el agrupamiento de los KIS en categorías, por lo que es razonable suponer que buena parte de las variaciones espaciales de su localización se asocian fuertemente con las ventajas y las dificultades de los territorios para crear, utilizar y difundir el conocimiento que se incorpora en la producción de dichos servicios.

La heterogeneidad de funciones y modos de producción es un rasgo peculiar de estos servicios. Más que un "sector» o un "clúster» de actividades, los KIS están presentes en casi todas las industrias (OECD, 2006: 31; Consoli y Elche, 2009) y, a menudo, conectan diversos sectores productivos entre sí y con el consumo. Entre estas funciones, destacan las de adaptación, difusión de innovaciones y organización de recursos para el desarrollo del conocimiento (Miles, 2003; Kemppilä y Mettänen, 2004; OECD, 2006; Wood, 2006; European Commisison, 2007; Albors-Garrigos et al., 2009; Doloreux et al., 2010; Rodríguez y Camacho, 2010), así como su contribución al desarrollo regional (Herstad y Eberbersger, 2015).

Este anclaje de los KIS en múltiples partes del sistema socioeconómico, así como la sincronía de producción y consumo de los servicios, genera múltiples formas de producción: por empresas pequeñas, medianas y grandes; por diversas instituciones; orientados al consumidor final o a las empresas. Esta variedad provoca que, en la localización de los KIS, algunos aspectos cobren mayor importancia que en otras actividades del sector industrial dedicadas a la investigación y desarrollo y a la producción del conocimiento.

Cabe añadir también que algunos servicios — sanitarios, educativos, sociales y culturales - forman parte del conjunto de derechos de los ciudadanos de los países desarrollados, por lo que la Administración debe asegurar su prestación, de forma directa o indirecta. Este hecho condiciona los patrones de localización de estos KIS, que tienden a reproducir la distribución espacial de la población.

La elección de la localización de un servicio intensivo en conocimiento expresa las oportunidades que, para un agente económico, reúne un territorio 


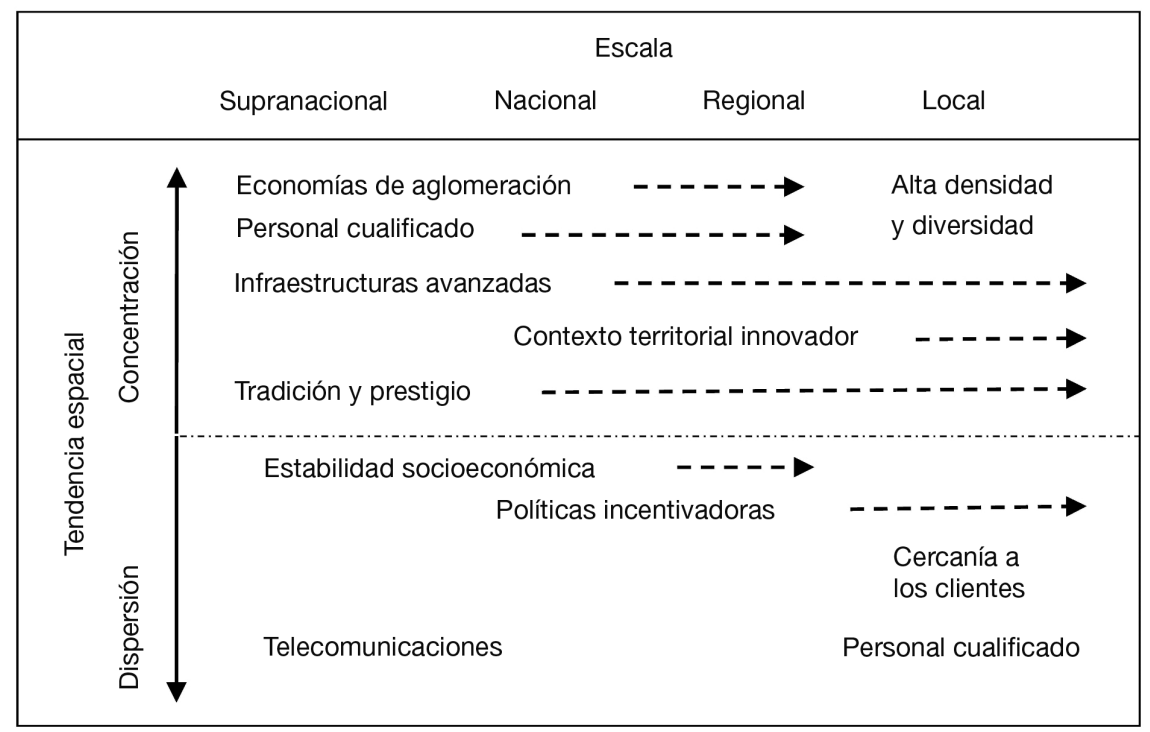

Figura 1. Algunos factores de localización de los KIS según su escala de actuación y tendencias espaciales.

Fuente: elaboración propia.

para el desarrollo de cierta actividad. La localización no es un mero soporte pasivo o un contenedor, sino un componente activo de la totalidad de operaciones realizadas que sintetiza bien la influencia que ese lugar ejerce acerca de un conjunto de condiciones sociales, económicas, tecnológicas, etc. que favorecen o inhiben la creación de KIS.

La investigación empírica sobre la localización de los KIS —y de los servicios en general - ha destacado la importancia del componente humano (cualificación, capacidad de comunicación y relación interpersonal), sobre todo en el desarrollo de los KIS que incorporan tecnología y conocimiento avanzados (actividades de investigación y desarrollo, programación informática, consultoría especializada) que emplean a personal muy cualificado de formación diversa (ingeniería, informática, arquitectura, economía, derecho, etc.), con capacidad para interpretar información y resolver problemas (Tether y Hipp, 2001; Miles, 2008: 5).

Por otro lado, Merino y Rubalcaba (2013) han identificado una compleja malla de factores interrelacionados, como la distancia al mercado, los costes de coordinación, la necesidad de proximidad y de elevada interacción, la emergencia de regiones competitivas, etc., que influyen de forma diferenciada en la localización de cada tipo de KIS. De este enfoque, se desprende que lo más relevante desde el punto de vista de la localización de los KIS reside en la acción conjunta de distintos factores, lo que da lugar a matrices de condiciones diferentes en cada territorio que fundamentan el grado desigual de especialización, concentración y dispersión espacial de los KIS (figura 1). 
Los KIS, especialmente los que utilizan más intensidad de conocimiento e información (investigación y desarrollo, programación informática y otras), se localizan en áreas innovadoras — creative milieux — de diverso alcance espacial (local, metropolitano o regional), según el tamaño y las estrategias de las empresas para aprovechar las externalidades. Los KIS con menor intensidad de conocimiento y orientados al mercado tienden a dispersarse, aunque de forma selectiva. Existen, pues, rasgos de localización propios de las distintas categorías de KIS que se resumen en la tabla siguiente.

Tabla 1. Tendencias locacionales de los servicios intensivos en conocimiento (KIS)

\begin{tabular}{|c|c|c|}
\hline $\begin{array}{l}\text { Tipo de servicio intensivo } \\
\text { en conocimiento (KIS) }\end{array}$ & Tendencias locacionales & $\begin{array}{l}\text { Algunas referencias } \\
\text { bibliográficas }\end{array}$ \\
\hline $\begin{array}{l}\text { KIS con uso intensivo } \\
\text { de conocimiento y } \\
\text { tecnología avanzada. }\end{array}$ & $\begin{array}{l}\text { Concentración y aglomeración } \\
\text { generalmente más acentuadas } \\
\text { que los de otras actividades } \\
\text { económicas a todas las escalas. } \\
\text { El grado de concentración y de } \\
\text { dispersión se relaciona con el } \\
\text { nivel de desarrollo de los países. }\end{array}$ & $\begin{array}{l}\text { Camacho et al. (2012); } \\
\text { European Commision (2012); } \\
\text { Merino y Rubalcaba (2013). }\end{array}$ \\
\hline
\end{tabular}

Servicios con uso intensivo de conocimiento orientados al mercado y a las empresas (excepto servicios de intermediación financiera).
Elevada concentración espacial a todas las escalas, especialmente los servicios avanzados a las empresas. Los avances de las telecomunicaciones han impulsado procesos de dispersión de actividades rutinarias y de concentración de las tareas de dirección.

Servicios financieros con uso Las nuevas tendencias intensivo de conocimiento.

locacionales adquieren una bifurcación nítida a todas las escalas: las sedes centrales y las funciones de dirección se especializan y las oficinas con tareas administrativas y de comercialización se dispersan de forma selectiva por el espacio urbano para acercarse a la clientela potencial.

Otros servicios intensivos en conocimiento.

$$
\begin{aligned}
& \text { Los servicios de este grupo } \\
& \text { (sanitarios, educativos, } \\
& \text { culturales) utilizan conocimiento } \\
& \text { y tecnologías avanzadas, pero } \\
& \text { cuando son prestados por insti- } \\
& \text { tuciones públicas su localización } \\
& \text { tiende a seguir los patrones } \\
& \text { espaciales de la población con } \\
& \text { cierto grado de concentración de } \\
& \text { las funciones más especializadas. }
\end{aligned}
$$

\author{
Daniels y Moulaert (1991); \\ Coffey y Bailly (1992); Bailly \\ y Coffey (1994); Moulaert et \\ al. (1997); Aguiléra (2003); \\ Taylor \\ et al. (2014).
}

Xia et al. (2007);

Capelle-Blancard y Tadjedine (2009); Alonso et al. (2014).

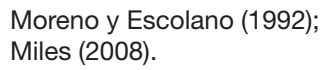

Fuente: elaboración propia. 
Los resultados de las investigaciones sugieren que la intensidad de conocimiento invertida en la producción de un KIS propende a generar estructuras espaciales polarizadas a todas las escalas. Los KIS con elevada intensidad de conocimiento y tecnología tienden a aglomerarse en las ciudades mundiales (Sassen, 1991), en grandes centros urbanos de regiones y países que forman parte de redes urbanas globales o regionales (Krätke, 2007). A escala intraurbana, los patrones espaciales son complejos y presentan agrupaciones más o menos compactas en los centros y en las periferias de las ciudades, en función de la estructura urbana y la localización de equipamientos y recursos, como parques tecnológicos, universidades, etc. (Halbert y Pain, 2009).

En general, todos los razonamientos y los factores aportados para explicar la distribución espacial de los KIS se relacionan con las oportunidades asociadas a las economías de aglomeración, enmarcadas en los procesos de integración regional y mundialización. Ello no excluye que determinados factores puedan concurrir en otras áreas no urbanas para generar entornos territoriales innovadores que favorezcan el desarrollo de los KIS (Doloreux et al., 2008).

Por otro lado, el desenvolvimiento de los KIS suele requerir de infraestructuras técnicas avanzadas (telecomunicaciones, laboratorios, universidades) (Van de Vijver et al., 2014), en ocasiones solo disponibles en las grandes ciudades, que también suelen contar con un rico patrimonio cultural y con un prestigio nacional o internacional.

En consecuencia, las ventajas que poseen las grandes ciudades las convierten en el locus privilegiado para la localización de los KIS, ya que en estos espacios se pueden obtener retornos crecientes. Los avances en las telecomunicaciones han propiciado la dispersión espacial de algunos tipos de KIS, pero esta ha sido de alcance limitado y muy selectiva, en todo caso menor que lo técnicamente posible. La difusión de Internet y de las redes de telecomunicaciones ha acentuado, incluso, la función de las ciudades como centros de difusión de externalidades (Bade et al., 2004: 29) y como motores de la desmaterialización de la producción (Halbert, 2005).

En este trabajo, se asume la hipótesis de que la concentración espacial de los KIS es una función escalar de la intensidad de conocimiento utilizado en la producción de servicio. Esta formulación general implica lo siguiente:

a) Que la intensidad per cápita de KIS intensivos en conocimiento y tecnología es creciente a medida que aumenta el tamaño demográfico (y seguramente económico) de las unidades espaciales en las que se localizan.

b) Que la función que relaciona la proporción de KIS con el tamaño demográfico de las unidades espaciales tiene una pendiente distinta para cada grupo de KIS, más pronunciada cuanto mayor es la intensidad de conocimiento y de tecnologías invertidas en el servicio.

c) Los patrones espaciales de los KIS presentan un grado más elevado de concentración y aglomeración que los de la población. 


\section{Metodología y datos}

Para delimitar las actividades KIS y agruparlas en categorías de intensidad de conocimiento y tecnología utilizados, así como la orientación al mercado final o a las empresas, se ha seguido la clasificación de EUROSTAT (s/f-b) que distingue cuatro categorías de KIS formadas por actividades definidas a dos dígitos de la NACE rev. 2 (tabla 2). Esta clasificación difiere de la anterior (NACE rev. 1.1.), especialmente el grupo de los KIS con uso de tecnología avanzada, que incorpora nuevas ramas.

La magnitud de cada actividad se ha medido por el número de personas afiliadas a la Seguridad Social el 31 de diciembre de 2011 en cada municipio, clasificadas en ramas de actividad de la CNAE rev. 2 con dos dígitos (NACE). La persona es una unidad funcional más homogénea que la unidad empresa y de uso muy generalizado en los estudios empíricos. Los datos recogen a las personas que trabajan por cuenta propia y ajena, tanto en las empresas como en las instituciones. La población analizada es la del censo de 2011 (31 de marzo).

Tabla 2. Categorías de servicios con uso intensivo de conocimiento y actividades definidas a dos dígitos de la NACE

\begin{tabular}{|c|c|}
\hline Grupos & Ramas de actividad definidas a dos dígitos (NACE rev. 2) \\
\hline $\begin{array}{l}\text { 1. Servicios con uso } \\
\text { intensivo de conoci- } \\
\text { miento y tecnología } \\
\text { avanzada. }\end{array}$ & $\begin{array}{l}\text { 59: Actividades cinematográficas, de vídeo y de programas de } \\
\text { televisión, grabación de sonido y edición musical; } 60 \text { : Actividades } \\
\text { de programación y emisión de radio y televisión; } 61 \text { : Telecomunica- } \\
\text { ciones; } 62 \text { : Programación informática, consultoría y otras activida- } \\
\text { des relacionadas con la informática; } 63 \text { : Servicios de información; } \\
\text { 72: Investigación y desarrollo. }\end{array}$ \\
\hline $\begin{array}{l}\text { 2. Servicios con uso inten- } \\
\text { sivo de conocimiento } \\
\text { orientados al mercado y } \\
\text { a las empresas (excepto } \\
\text { servicios de intermedia- } \\
\text { ción financiera). }\end{array}$ & $\begin{array}{l}\text { 50: Transporte marítimo y por vías navegables interiores; 51: } \\
\text { Transporte aéreo; } 69 \text { : Actividades jurídicas y de contabilidad; } \\
\text { 70: Actividades de las sedes centrales y actividades de consultoría } \\
\text { de gestión empresarial; 71: Servicios técnicos de arquitectura e } \\
\text { ingeniería, ensayos y análisis técnicos; } 73 \text { : Publicidad y estudios } \\
\text { de mercado; 74: Otras actividades profesionales, científicas y } \\
\text { técnicas; 78: Actividades relacionadas con el empleo; } 80 \text { : Activida- } \\
\text { des de seguridad e investigación. }\end{array}$ \\
\hline $\begin{array}{l}\text { 3. Servicios financieros } \\
\text { con uso intensivo de } \\
\text { conocimiento. }\end{array}$ & $\begin{array}{l}\text { 64: Servicios financieros, excepto seguros y fondos de pensiones; } \\
\text { 65: Seguros, reaseguros y fondos de pensiones, excepto Segu- } \\
\text { ridad Social obligatoria; } 66 \text { : Actividades auxiliares a los servicios } \\
\text { financieros y a los seguros. }\end{array}$ \\
\hline $\begin{array}{l}\text { 4. Otros servicios intensi- } \\
\text { vos en conocimiento. }\end{array}$ & $\begin{array}{l}\text { 58: Actividades de edición; 75: Actividades veterinarias; } \\
\text { 84: Administración pública y defensa; 85: Educación; 86: Activida- } \\
\text { des sanitarias; 87: Asistencia en establecimientos residenciales; } \\
\text { 88: Actividades de servicios sociales sin alojamiento; 90: Activida- } \\
\text { des de creación, artísticas y de espectáculos; } 91 \text { : Actividades } \\
\text { de bibliotecas, archivos, museos y otras acciones culturales; } \\
\text { 92: Actividades de juegos de azar y apuestas; 93: Actividades } \\
\text { deportivas, recreativas y de entretenimiento. }\end{array}$ \\
\hline
\end{tabular}

Fuente: EUROSTAT (s/f-b). 
Las unidades espaciales del estudio tienen dos niveles de resolución. Por una parte, se han utilizado las áreas funcionales urbanas (FUA: Functional Urban Areas) del proyecto ESPON (2013). Las FUA españolas son delimitaciones espaciales bastante coherentes con el funcionamiento socioeconómico del territorio, a pesar de las considerables diferencias en extensión, población, volumen, economía, etc. que presentan. Las unidades espaciales funcionales son más adecuadas que las administrativas para el estudio de la localización de actividades económicas, por lo que, en la medida de lo posible, se ha preferido su uso para este fin (Ruiz-Valenzuela et al., 2006; Davi et al., 2009). En este trabajo, las FUA se han utilizado para valorar la especialización y la concentración espacial de los KIS.

Por otra, se han usado los 8.102 municipios con datos válidos que permiten precisar los patrones espaciales de las actividades y medir la posible aglomeración de las mismas.

La investigación empírica de este trabajo se desarrolla en torno a los conceptos de concentración, especialización y aglomeración, estudiados a dos escalas distintas: área funcional urbana y municipio. Examinamos, en primer lugar, el grado de concentración de los KIS entre todas las áreas funcionales urbanas. La concentración espacial es una noción que se refiere al grado de homogeneidad con que se reparte una variable entre las unidades espaciales del área de estudio. El índice de Gini es, con mucha seguridad, la técnica más empleada para analizar la concentración espacial. Sin embargo, no es adecuado cuando las unidades espaciales son muy diferentes, como es el caso de las áreas funcionales y los municipios. Por esta razón, se ha preferido utilizar un índice ajustado de concentración geográfica (CGA) propuesto por Spiezia (2003), ya que pondera los valores de la variable con el tamaño demográfico (o espacial) de las unidades. Su desarrollo es como sigue:

$$
C G=\sum_{i=1}^{N}\left|y_{i}-a_{i}\right|
$$

Donde: CG es una medida absoluta de concentración geográfica; | indica el valor absoluto; $y_{\mathrm{i}}$ es la proporción de la variable $y$ en la unidad espacial $i$ respecto del total del área de estudio; $a_{i}$ representa la proporción de población o superficie de la unidad espacial $i$ respecto del total del área de estudio.

El índice alcanza su valor máximo cuando toda la variable se concentra en la unidad más pequeña de la zona de estudio:

$$
C G^{M A X}=\sum_{i \approx \min } a_{i}+1-a_{\min }=1+1-2 a_{\min }=2\left(1-a_{\min }\right)
$$

Donde $a_{\min }$ : peso relativo de la unidad más pequeña (población o superficie).

El índice se puede normalizar para que sus valores se sitúen en el rango 0 (ausencia de concentración) a 1 (máxima concentración):

$$
\mathrm{CGA}=\mathrm{GC} / \mathrm{GC}^{\mathrm{MAX}}
$$


En una etapa posterior del análisis, tratamos de establecer el nivel de especialización de las áreas funcionales urbanas en cada uno de los tipos de servicios intensivos en conocimiento. Para ello, se ha utilizado el coeficiente de localización $(\mathrm{QL})$, cuya razón matemática se expresa de la manera siguiente:

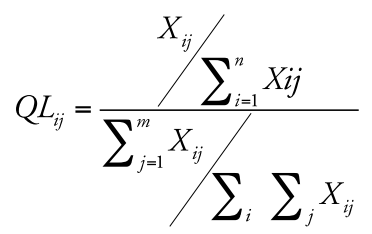

Coeficiente de localización de la actividad $j$ en la ciudad $i\left(Q L_{i j}\right)$ :

$X_{i j}$ : Ocupados en la actividad $i$ en la ciudad $j$.

$\sum_{i=1}^{n} X_{i j}$ Ocupados en todas las actividades de la ciudad $j$.

$\sum_{j=1}^{m} X_{i j^{j}}$ Ocupados en la actividad $i$ en todas las ciudades.

$\sum_{i} \sum_{j} X_{i j^{*}}$ Ocupados en todas las actividades y en todas las ciudades.

El valor de coeficiente de localización es siempre positivo. Cuando supera la unidad en un tipo de actividad y en un lugar, se puede considerar que existe cierto grado de especialización en tal actividad y en tal lugar. En este trabajo, se ha considerado que un área funcional tiende a especializarse cuando el valor de su QL en algún tipo de KIS supera el 1,1; en cambio, otros estudios han aplicado un umbral de 1,25 (Miller et al., 2001) o aún más elevados de 3 (Isaksen, 1996). Los criterios para establecer este límite dependen de la variabilidad y de los valores máximos del coeficiente, pero no se aplica un único umbral (Martin y Sunley, 2003; O’Donoghe y Gleave, 2004).

El estudio de la localización de los KIS en las diferentes áreas urbanas se completa mediante el análisis de la relación funcional entre la población ocupada en KIS ( $Y$ ) y el tamaño demográfico de las FUA $(X)$. Esta relación se ha estimado mediante el ajuste de una función potencial $Y=\mathrm{a}_{0} X^{X}$, en la que el valor del exponente $\beta$ recoge las múltiples interacciones entre los KIS y la totalidad socioeconómica de un área, representada por su población.

La segunda parte del análisis se centra en la escala municipal, ya que se refiere a los 8.102 municipios que integran las áreas funcionales urbanas. El nivel de concentración de los servicios estudiados y la especialización municipal resultante se establecen mediante los índices ya explicados. Además, abordamos el análisis de su aglomeración espacial, concepto más amplio que los anteriores, pues combina la concentración de los valores de una variable con la proximidad (o lejanía) de las unidades en las que se concentran. Para estimar la aglomeración espacial y delimitar clústeres espaciales de actividades, utilizamos el índice $I$ de Moran (global), que se calcula del modo siguiente (O'Sullivan y Unwin, 2010: 205-222): 


$$
I=\frac{N}{\sum_{i}\left(y_{i}-\bar{y}\right)^{2}} \frac{\sum_{i} \sum_{j} w_{i j}\left(y_{i}-\bar{y}\right)\left(y_{j}-\bar{y}\right)}{\sum_{i=1}^{n} \sum_{j=1}^{n} w_{i j}}
$$

Donde $N$ es el número de unidades espaciales indexadas por $i$ y $j ; y$ es la variable estudiada; $\bar{y}$ es la media de $y, \mathrm{y} w_{i j}$ es un elemento de la matriz de ponderaciones espaciales. Los valores de $I$ mayores que los esperados indican autocorrelación espacial positiva de la localización de los valores de una variable (por ejemplo, ocupados en las actividades KIS), mientras que los valores de $I$ inferiores a los esperados indican autocorrelación espacial negativa.

La versión local del índice $I$ de Moran permite calcular la autocorrelación espacial de los valores de la variable en cada localización y con medidas de significación estadística. El índice $I_{i}$ local de Moran para cada observación $i$ se obtiene mediante la fórmula siguiente:

$$
I_{i=} z_{i} \sum w_{i j} z_{j}
$$

Donde $z_{i}$ representa las puntuaciones $z$ de la variable de estudio. Los valores positivos de $I_{i}$ se producen cuando los valores altos o bajos de la variable de interés están unos cerca de otros; los valores negativos de $I_{i}$ se originan cuando valores altos y valores bajos de la variable se localizan en la misma área. Ambos índices se han calculado mediante el programa Geoda. La vecindad de un municipio se ha delimitado con el criterio del «movimiento de la reina del ajedrez», es decir: son vecinos de un municipio aquellos que comparten con en él, al menos, un vértice del perímetro que define sus límites territoriales. Este procedimiento asegura que la mayor parte de los municipios tenga al menos otros ocho vecinos, número mínimo necesario para garantizar la validez de los resultados en distribuciones muy sesgadas, como es el caso. En la tabla 3, se resume el proceso metodológico seguido y los datos empleados en este trabajo.

\begin{tabular}{|c|c|c|}
\hline $\begin{array}{l}\text { Unidades espaciales } \\
\text { de análisis }\end{array}$ & Tipo de análisis & Índices \\
\hline $\begin{array}{l}\text { Áreas funcionales } \\
\text { (FUA) (189, no conti- } \\
\text { guas). }\end{array}$ & $\begin{array}{l}\text { Concentración interFUA e intraFUA. } \\
\text { Especialización. } \\
\text { Relación funcional con el tamaño } \\
\text { demográfico. }\end{array}$ & $\begin{array}{l}\text { Índice ajustado de concentración } \\
\text { geográfica (CGA). } \\
\text { Coeficientes de localización (QL). } \\
\text { Coeficiente de ajuste de una } \\
\text { función potencial (población y } \\
\text { número de afiliados). }\end{array}$ \\
\hline $\begin{array}{l}\text { Municipios ( } 8.102 \text { con } \\
\text { datos válidos). }\end{array}$ & $\begin{array}{l}\text { Concentración y aglomeración. } \\
\text { Especialización. }\end{array}$ & $\begin{array}{l}\text { CGA; índice } / \text { de Moran; } I_{i} \\
\text { de Moran local I. } \\
\text { Coeficientes de localización (QL). }\end{array}$ \\
\hline
\end{tabular}

Tabla 3. Resumen de los datos y de los procedimientos analíticos utilizados

Fuente: elaboración propia. 


\section{Los contrastes espaciales de los KIS en España: alcance de la escala y de la intensidad de conocimiento}

El peso relativo de las actividades de la economía del conocimiento en España, medido por la población ocupada, es menor que el promedio de las mismas en los países de la Unión Europea, lo que constituye un importante rasgo que diferencia el modelo de la economía española de los modelos de las economías más avanzadas.

No obstante, a pesar de los avatares de la economía española desde 2008, la marcha de la economía del conocimiento muestra una tendencia sostenida al crecimiento, aunque a un ritmo lento que retarda la convergencia con los modelos económicos basados en el conocimiento, la creatividad y la innovación tecnológica. En el sexenio 2008-2013, el ascenso del empleo de la economía del conocimiento se ha registrado exclusivamente en los KIS, mientras que han retrocedido las manufacturas de tecnología media y avanzada. En España, el empleo en los KIS ha pasado del 30,6\% en 2008 al 35,9\% en 2013, mientras en el mismo período el empleo en manufacturas de elevada y media tecnología ha descendido del 4,1\% al 3,7\% (EUROSTAT, s/f-a). Es probable que buena parte de la actividad se haya externalizado desde los sectores manufactureros hasta empresas de servicios (outsourcing).

La tabla 4 contiene datos más detallados que permiten valorar mejor el significado económico, social y espacial de los KIS en España.

Por una parte, el mayor volumen de ocupación corresponde al grupo «otros servicios», entre los que sobresalen los relacionados con el «estado del bienestar» (sociales, sanitarios, educativos, culturales), que se dirigen directamente a mejorar las condiciones de vida de las personas. En la producción de algunos de estos servicios, se invierte una elevada intensidad de conocimiento y tecnología avanzada, como en los sanitarios y educativos, pero su provisión, en su mayor parte pública, hace que tengan condiciones de desarrollo y localización particulares. Por supuesto, la dinámica de los KIS públicos impulsa o frena el crecimiento de las restantes actividades KIS privadas.

Tabla 4. Afiliados en los KIS por grupos de actividad y tamaño demográfico de los municipios

\begin{tabular}{lrrrr}
\hline & \multicolumn{2}{c}{ Intervalos de población 2011 (habitantes) } & Total \\
\cline { 2 - 4 } \multicolumn{1}{c}{ Grupo de KIS } & Hasta 2.000 & $2.001-10.000$ & Más de 10.000 & Try \\
\hline KIS con elevada intensidad tecnológica & 2.571 & 18.484 & 402.798 & 423.853 \\
KIS orientados al mercado y a las empresas & 9.998 & 52.273 & 927.079 & 989.350 \\
KIS: servicios financieros & 2.023 & 1.560 & 391.144 & 394.727 \\
KIS: otros servicios & 75.065 & 225.266 & 3.108 .225 & 3408.556 \\
Total KIS & 89.657 & 297.583 & 4.829 .246 & 5.216 .486 \\
Total ocupados & 713.655 & 2.013 .042 & 13445550 & 16.172 .247 \\
Total KIS/ total ocupados (\%) & 12,6 & 14,8 & 35,9 & 32,3 \\
Número de municipios & 5.766 & 1.560 & 759 & 8.085 \\
Porcentaje de población & 6,0 & 15,0 & 79,1 & 100,0 \\
\hline
\end{tabular}

Fuente: Tesorería General de la Seguridad Social e Instituto Nacional de Estadística. 
Por otra, se evidencia la concentración creciente de los KIS a medida que aumenta el tamaño demográfico de los municipios, aunque el grado de la misma difiere según el tipo de KIS, lo que origina patrones espaciales de concentración, aglomeración y especialización complejos que se estudian a continuación.

\subsection{Concentración y especialización de los KIS en las áreas funcionales (FUA)}

La característica general más destacable de la distribución espacial de los KIS en España, obvia por lo demás, es su marcada concentración a todas las escalas, igual que otras actividades de la economía del conocimiento (Méndez, 2013: 29).

Esta afirmación debe matizarse con dos notas significativas: una se refiere a que la concentración también es acusada cuando se mide con unidades funcionales y no solo administrativas, arbitrarias hasta cierto punto; la otra da cuenta de que la concentración espacial de los KIS es mayor que la de la población.

En la tabla 5, se observa que las seis áreas funcionales con más de 100.000 afiliados a la Seguridad Social en actividades de los KIS reúnen solo algo menos de la mitad de los afiliados en los KIS en España, frente al 35\% de la población y una proporción de superficie aún menor, que no llega al 10\%. Estos valores prácticamente se invierten para el caso del territorio «rural», que no forma parte de las áreas funcionales urbanas, pues este ocupa más de la mitad de la superficie pero solo acumula el 18\% de la población y el 6,5\% de los ocupados en los KIS.

La fuerte concentración se debe, en buena parte, al peso de un área funcional muy grande, Madrid, donde se localiza más de la quinta parte de los ocupados en los KIS, conectada a otras menores, contiguas o no (Ávila, Segovia, Toledo,

Tabla 5. Afiliados en los distintos grupos de KIS en las áreas funcionales con más de 100.000 afiliados a la Seguridad Social

\begin{tabular}{|c|c|c|c|c|c|c|c|}
\hline \multirow[b]{2}{*}{ FUA } & \multirow{2}{*}{$\begin{array}{c}\text { Población } 11 \\
(000)\end{array}$} & \multirow{2}{*}{$\begin{array}{l}\text { Superficie } \\
\mathrm{km}^{2}(000)\end{array}$} & \multicolumn{5}{|c|}{ Afiliados en KIS (000) } \\
\hline & & & IT & ME & SF & OT & Total \\
\hline Madrid & 6.728 & 13,8 & 173,7 & 288,8 & 110,2 & 551,4 & 1124,2 \\
\hline Barcelona & 4.595 & 2,9 & 68,4 & 146,6 & 54,6 & 413,1 & 682,7 \\
\hline Valencia & 1.774 & 4,6 & 16,0 & 41,5 & 19,1 & 139,9 & 216,5 \\
\hline Sevilla & 1.404 & 3,9 & 16,2 & 34,5 & 12,9 & 122,2 & 185,7 \\
\hline Bilbao & 1.044 & 2,2 & 14,8 & 29,5 & 10,6 & 94,7 & 149,7 \\
\hline Zaragoza & 876 & 15.1 & 8,0 & 16,9 & 8,8 & 73,2 & 106,9 \\
\hline Total 6 & 18.922 & 42,5 & 316,0 & 612,3 & 249,3 & $1.625,0$ & $2.803,2$ \\
\hline España & 47.157 & 506,5 & 423,8 & 989,3 & 394,7 & $3.408,5$ & $5.216,5$ \\
\hline Total 6 / España (\%) & 34,8 & 8,4 & 70,1 & 56,4 & 54,7 & 40,9 & 47,3 \\
\hline Rural / total & 17,9 & 56,7 & 2,4 & 5,1 & 3,1 & 7,8 & 6,5 \\
\hline \multicolumn{3}{|c|}{ Índice de concentración CGA } & 0,37 & 0,25 & 0,30 & 0,18 & 0,20 \\
\hline
\end{tabular}

IT: elevada intensidad de conocimiento y tecnología; ME: orientados al mercado y a las empresas; SF: servicios financieros; OT: otros servicios.

Fuente: Tesorería General de la Seguridad Social e Instituto Nacional de Estadística. 


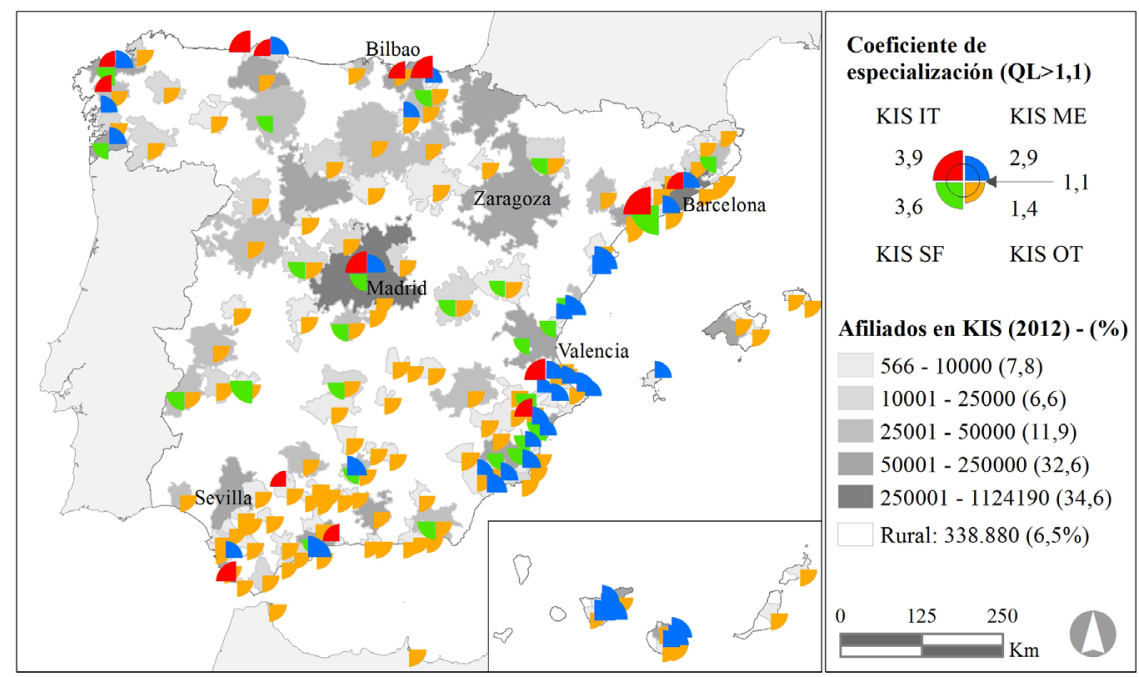

Figura 2. Coeficientes de especialización (QL) de los tipos de servicios y total de afiliados en KIS en las áreas funcionales.

Fuente: Tesorería General de la Seguridad Social.

Ciudad Real, etc.) y rodeada por una extensa zona de valores muy débiles. Las áreas litorales, en cambio, se caracterizan por la presencia de conglomerados de áreas funcionales grandes, medianas y pequeñas que orlan la costa mediterránea (Cataluña, Comunidad Valenciana y, en menor medida, Andalucía) y atlántica (País Vasco y Galicia), como refleja el mapa de la figura 2.

Como era de esperar, el grado de concentración espacial varía para cada categoría de servicios. Los KIS con «elevada intensidad de conocimiento y tecnología avanzada" son los que tienen el índice de concentración más alto (CGA $=0,37$ ), seguidos de los «servicios financieros», los «orientados al mercado y a las empresas» $y$, finalmente, de los «demás servicios».

La composición interna por tipos de KIS en las áreas funcionales presenta variaciones relacionadas, principalmente, con su propio tamaño y especialización y con estas mismas propiedades en las áreas contiguas y cercanas. En las FUA más pequeñas, suele tener un peso considerable el KIS «otros servicios», en tanto que en las más grandes la estructura es más equilibrada, incluso aunque puedan tener algún tipo de especialización.

La especialización de las áreas funcionales conforma un patrón a escala nacional muy bien definido, que resulta, por un lado, de la fuerte concentración más o menos selectiva de los diferentes grupos de KIS y, por otro, de la aglomeración espacial de los diferentes tipos de especialización (figura 2).

Los valores de especialización más bajos $(\mathrm{QL}$ máximo $=1,4)$ corresponden al grupo "otros servicios» y son los más dispersos espacialmente. Las FUA especializadas en «servicios financieros» son menos frecuentes y sus índices de 
especialización, algo más elevados $(\mathrm{QL}$ máximo $=3,6)$ y tienden también a la dispersión territorial. En cambio, la especialización en servicios «orientados al mercado y a las empresas» aparece de forma más selectiva en las FUA del litoral o de las islas (QL máximo = 2,9). La especialización en KIS con elevada intensidad de conocimiento solo se produce en unas cuantas FUA, y sus valores de especialización son más elevados $(\mathrm{QL}$ máximo $=3,9)$.

Así pues, la intensidad de conocimiento utilizada en la producción de los servicios y el tipo de regulación de los mismos se asocian al grado de especialización y concentración espacial. Estas dos últimas también son variables interrelacionadas.

El examen del perfil de especialización de cada FUA revela la existencia de zonas bien diferenciadas en cuanto a la estructura espacial y funcional de los KIS. Por una parte, destaca la FUA de Madrid en el centro peninsular, de gran tamaño y diversificada. Por otra, aparecen grupos de áreas de tamaño mediano y pequeño especializadas en uno o dos tipos de KIS, pero próximas entre sí, de tal manera que funcionan como una unidad potente y variada; se localizan en Cataluña, Comunidad Valenciana y País Vasco y, con dimensiones menores, también en Andalucía y Galicia. Finalmente, en el resto del territorio, la especialización en «otros servicios» o en "servicios financieros» tiene un alcance regional o local.

Las variaciones espaciales sistemáticas de la concentración y de la especialización son un buen indicio de la actuación consistente de factores como la intensidad de conocimiento y la orientación al mercado final o intermedio, así como de la influencia de la organización espacial y funcional de la red urbana. A continuación, se analizan estos aspectos con una resolución de mayor detalle.

\subsection{Concentración, aglomeración y especialización de los KIS a escala municipal}

La concentración espacial de los KIS es extraordinariamente elevada cuando se mide con unidades espaciales de gran resolución como los municipios. En este caso, los contrastes se incrementan, ya que, en una fracción insignificante del territorio — no llega al 0,5\%—, se localiza un tercio de los KIS y algo más del $16 \%$ de la población.

Los valores globales deben ser desglosados, ya que resultan de promediar diferentes categorías de KIS. En la tabla 6, se recoge el índice de concentración geográfica ajustado (CGA), cuyos valores son muy altos en los servicios con «elevada intensidad de conocimiento» y en los «servicios financieros», $\mathrm{y}$ bastante más bajos en los KIS «orientados al mercado y a las empresas» y en los «otros servicios». Ello se debe a que las actividades dirigidas a satisfacer las necesidades de las personas se dispersan siguiendo la distribución espacial de la población (actividades deportivas y de ocio, las relacionadas con el empleo), y más aún si su provisión es pública (educativas, sanitarias, asistenciales).

Por otro lado, hay que subrayar la considerable proporción de KIS «intensivos en conocimiento" y «servicios financieros» que se localizan en los municipios más grandes, es decir, en Madrid y Barcelona, que los colocan a considerable distancia de los demás municipios. 
Tabla 6. Afiliados a la Seguridad Social en los distintos grupos de KIS en los seis municipios con mayor número de afiliados

\begin{tabular}{|c|c|c|c|c|c|c|c|}
\hline \multirow[b]{2}{*}{ Municipio } & \multirow{2}{*}{$\begin{array}{c}\text { Población } 11 \\
(000)\end{array}$} & \multirow{2}{*}{$\begin{array}{c}\text { Superficie } \\
\mathrm{km}^{2}\end{array}$} & \multicolumn{5}{|c|}{ Afiliados en KIS (000) } \\
\hline & & & IT & ME & SF & OT & Total \\
\hline Madrid & 3.265 & 604,5 & 116,9 & 221,9 & 87,0 & 373,1 & 798,9 \\
\hline Barcelona & 1.615 & 99,5 & 46,1 & 93,4 & 40,8 & 255,4 & 435,7 \\
\hline Valencia & 798 & 136,7 & 9,9 & 28,1 & 17,0 & 101,2 & 156,1 \\
\hline Sevilla & 703 & 141,4 & 10,6 & 26,2 & 11,6 & 95,9 & 144,3 \\
\hline Zaragoza & 674 & 973,7 & 7,8 & 15,7 & 8,5 & 65,7 & 97,7 \\
\hline Málaga & 568 & 395,0 & 5,6 & 16,8 & 8,8 & 61,9 & 93,6 \\
\hline Total 6 & 7.624 & $2.350,8$ & 196,9 & 402,1 & 173,7 & 953,2 & $1.726,3$ \\
\hline Total 6 / España (\%) & 16,2 & 0,46 & 46,5 & 40,6 & 44,1 & 28,0 & 33,1 \\
\hline \multicolumn{3}{|c|}{ Îndice de concentración CGA } & 0,51 & 0,38 & 0,52 & 0,32 & 0,34 \\
\hline
\end{tabular}

IT: elevada intensidad de conocimiento y tecnología; ME: orientados al mercado y a las empresas; SF: servicios financieros; OT: otros servicios.

Fuente: Tesorería General de la Seguridad Social.

Los valores del índice I global de Moran muestran que la distribución espacial de los KIS en España no es aleatoria y que existe autocorrelación espacial positiva en todos los tipos de KIS, es decir, que se concentran. Los valores calculados de $I$ son significativamente más elevados que los esperados si los KIS se localizasen de forma aleatoria. Los valores estandarizados del índice oscilan entre $z=4,2$ para los "otros servicios" y $z=46,2$ para los KIS con «elevada intensidad de conocimiento». Estos valores tan extremos se producen porque la distribución real es muy sesgada, ya que, en algunos tipos de KIS, casi el $60 \%$ de los municipios carece de afiliados.

El paso lógico siguiente consiste en averiguar cómo se compone el patrón espacial de los KIS y el de cada grupo de KIS. Los resultados de aplicar el índice $I_{i}$ local de Moran muestran una configuración espacial compleja, que se define por dos propiedades estructurales principales: su elevada concentración espacial y la localización selectiva de las especializaciones.

En primer lugar, los afiliados en las diferentes actividades de los KIS están presentes en algo más del 90\% de los municipios españoles, aunque la mayor parte de los KIS se concentra en unos cuantos municipios. Si los municipios con afiliados en los KIS por encima del promedio nacional son pocos, su número se reduce considerablemente si se incluye la condición de que además estén por encima del promedio de sus vecinos (clases alto-alto y alto-bajo del $I_{i}$ de Moran). Esta característica general presenta diferencias claras por tipos de KIS: las actividades «orientadas al mercado y a las empresas» y «otros servicios» aparecen más dispersas y menos concentradas que los KIS con «elevada intensidad de conocimiento" y los «servicios financieros». Estos últimos tienen un número de afiliados menor que los KIS con «elevada intensidad de conocimiento», pero están presentes en más municipios y, al mismo tiempo, su grado de concentración es mayor, lo que indica que la reestructuración del sector ha producido una considerable concentración (tabla 7). 
Tabla 7. Distribución de los municipios según la clasificación del índice $I_{i}$ local de Moran

\begin{tabular}{lccccc}
\hline & \multicolumn{5}{c}{ Municipios en las clases del índice $I_{i}$ local de Moran } \\
\cline { 2 - 6 } \multicolumn{1}{c}{ Tipo de KIS } & Alto-alto & Alto-bajo & Bajo-bajo & Bajo-alto & No significativo \\
\hline KIS con elevada intensidad tecnológica & 169 & 15 & 498 & 435 & 7.076 \\
KIS orientados al mercado y a empresas & 224 & 11 & 1.338 & 469 & 6.151 \\
KIS: servicios financieros & 88 & 14 & 934 & 643 & 6.514 \\
KIS: otros servicios & 234 & 15 & 1.558 & 568 & 5.818 \\
Total KIS & 227 & 14 & 1.608 & 518 & 5.826 \\
Población & 434 & 25 & 1.744 & 359 & 5.631 \\
\hline
\end{tabular}

Fuente: Tesorería General de la Seguridad Social.

Los municipios cuyo número de afiliados es mayor que el promedio nacional y que el de su vecindad (clase alto-alto) tienden a agruparse espacialmente, es decir, a aglomerarse. Los clústeres que forman son muy diferentes en tamaño y oscilan entre los dos municipios de los más pequeños y los agrupamientos más grandes de Madrid (31 municipios), Barcelona (28 municipios) y Murcia (12 municipios) (figura 3).

Los conglomerados espaciales de municipios varían en número y en tamaño según los tipos de KIS. Los agrupamientos son más numerosos en los servicios «orientados al mercado y a las empresas» y en los «otros servicios», y más raros en los «servicios financieros» y en los servicios con «elevada intensidad de conocimiento». En estos últimos, además, solo dos municipios de la clase alto-alto se localizan en el área rural, todos los demás están en el territorio de alguna área funcional urbana. La clase alto-bajo (valores más altos que el promedio general y el de sus vecinos) sigue pautas similares, pues solo cuatro municipios de esta clase están en áreas rurales. Se trata, en casi todos los casos, de ciudades intermedias, generalmente aisladas en entornos económicos deprimidos, como Calatayud (Zaragoza), Barbastro (Huesca) o Sarriá (Lugo) (figura 4).

La localización de los clústeres produce un modelo general de acusado contraste entre el centro y la periferia, congruente con la estructura espacial, demográfica y económica española y con la configuración espacial de algunas redes de infraestructuras, como la de alta velocidad ferroviaria. En el centro de gravedad de esta distribución se localiza el gran conglomerado de la Región Metropolitana de Madrid, mientras que la mayoría de los restantes agrupamientos se sitúa en áreas del litoral peninsular y en las islas, en sectores muy selectivos en función de los tipos de KIS (figuras 3 y 4 ).

Por otra parte, la medida de la especialización a escala municipal evidencia que el funcionamiento de los KIS rebasa los confines de los municipios para conformar áreas más extensas que actúan como unidades espaciales y funcionales.

Los municipios muy grandes, como Madrid, Barcelona y Bilbao, tienen una composición de KIS netamente diversificada, pues sus índices de especialización se sitúan entre $\mathrm{QL}>1,1$ y $\mathrm{QL}<1,5$, y, además, se especializan en 


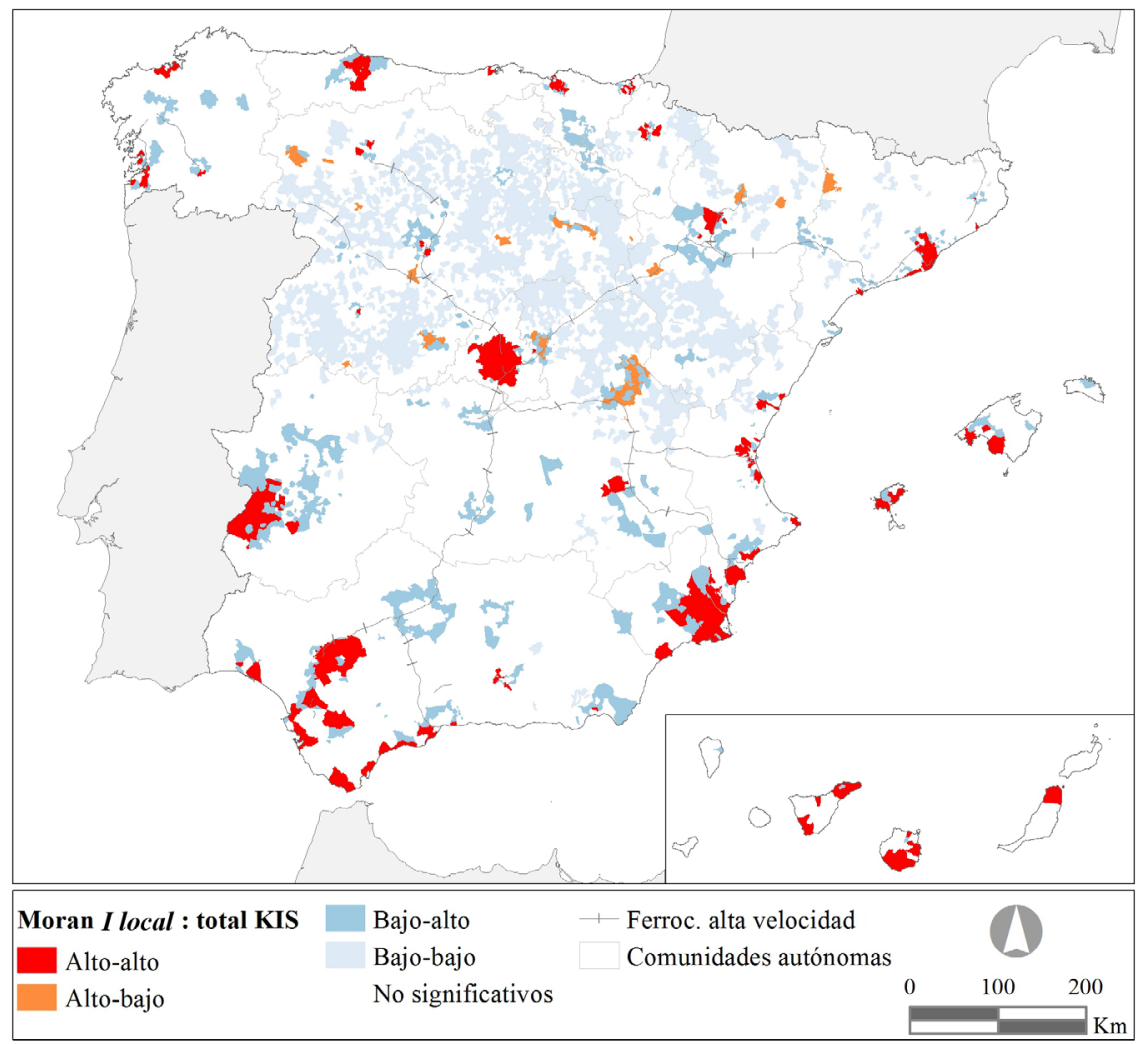

Figura 3. Clasificación del índice $I_{i}$ local de Moran de los municipios según el número de afiliados a la Seguridad Social en KIS.

Fuente: Tesorería General de la Seguridad Social.

«servicios intensivos en conocimiento», en "servicios financieros» y en servicios «orientados al mercado y a las empresas», pero no en "otros servicios». Otras grandes ciudades, como Valencia, Sevilla, Zaragoza, Palma de Mallorca, Alicante, Murcia, Vigo y otras presentan especialización, a veces acentuada, en servicios financieros $(1,1<\mathrm{QL}<1,7)$. En cambio, otros municipios de menor tamaño tienen valores muy altos de especialización en estos mismos tipos de KIS $(\mathrm{QL}>5)$, pero casi todos se localizan próximos a las grandes ciudades citadas.

La especialización destacada en "otros servicios», generada sobre todo por la presencia de equipamientos públicos (educativos, sanitarios, asistenciales), se corresponde con ciudades intermedias que, tradicionalmente, han actuado como centros de mercado de un área de influencia más o menos extensa (Huelva, Jaén, Cáceres, Pontevedra, Andújar, Teruel, Olivenza, etc.). 


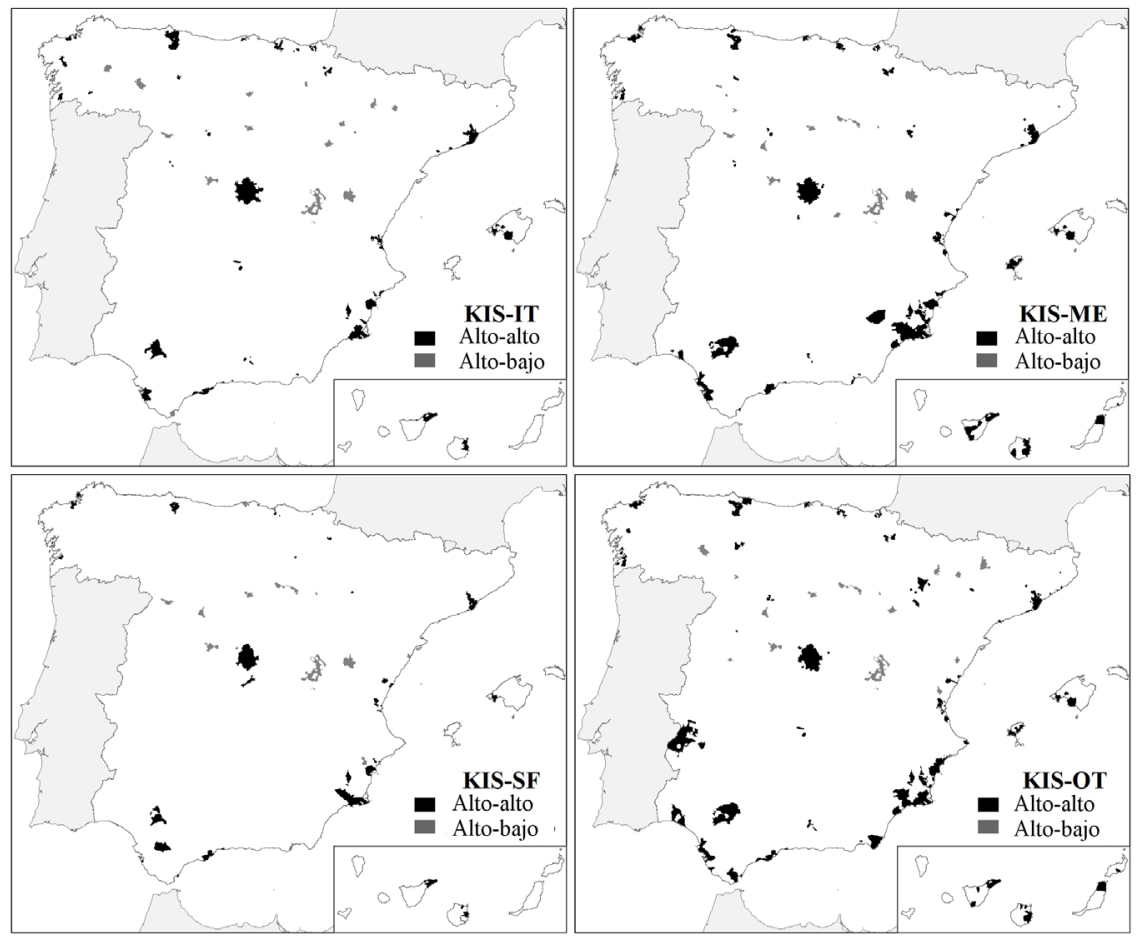

Figura 4. Clases alto-alto y alto-bajo del / local de Moran de los municipios según el número de afiliados a la Seguridad Social en los grupos de KIS.

IT: elevada intensidad de conocimiento y tecnología; ME: orientados al mercado y a las empresas; SF: servicios financieros; OT: otros servicios.

Fuente: Tesorería General de la Seguridad Social.

Finalmente, la especialización elevada de otros municipios pequeños obedece a circunstancias variadas, como la localización de universidades, de parques tecnológicos u otras, como en el caso de Canfranc, un pequeño municipio de la provincia de Huesca fronterizo con Francia, en donde, en el interior del túnel internacional del ferrocarril del mismo nombre, se encuentran las instalaciones del Laboratorio Subterráneo de Canfranc, causa del alto grado de especialización del municipio en KIS con «elevada intensidad de conocimiento».

Los resultados obtenidos son acordes con la tipología de inserción de las ciudades en la economía del conocimiento elaborada por Méndez et al. (2009) y con el funcionamiento espacial de las economías regionales (Salom et al., 2009). En general, la distribución espacial de los afiliados en los KIS por municipios presenta un grado de concentración espacial mayor que el de la población y se acentúa ostensiblemente si en vez de afiliados se consideran las empresas, sobre todo en los KIS con «elevada intensidad de conocimiento» $y$ 
en los «servicios financieros». Estos valores contrastan, en cierta medida, con la mayor dispersión por ciudades intermedias de las actividades de la economía del conocimiento del sector industrial.

\subsection{Relación entre los KIS y el tamaño demográfico de las FUA}

Los KIS son actividades muy importantes en los procesos de transformación social, pues impulsan cambios de variada naturaleza a través de la creación, la difusión y la aplicación del conocimiento, la innovación, la creatividad y la tecnología avanzada.

El desenvolvimiento de las empresas KIS es dificultoso por cuanto requiere de tareas precisas de coordinación y ensamblaje de elementos muy especializados — profesionales, infraestructuras y mercados-, tangibles e intangibles, algunos solo disponibles en determinados entornos territoriales. De aquí que, como se ha indicado, la localización sea un factor estratégico para el funcionamiento y el desarrollo de los KIS.

Ciertos componentes de los sistemas complejos, biológicos y sociales, como los sistemas urbanos o los sistemas de áreas funcionales, muestran relaciones escalares con el tamaño de las unidades, es decir, su proporción per cápita es creciente a medida que aumenta el tamaño de las entidades en las que se asientan (ciudades, áreas funcionales) (Bettencourt et al., 2007).

Esta asociación se mide a través del ajuste de una función potencial entre la población total $(X)$, que se utiliza como estimador del tamaño de cada FUA, y el número de afiliados a la Seguridad Social en cada grupo de KIS (Y). El valor del exponente de $\beta$ de la función condensa el carácter de la relación.

Los resultados más importantes se recogen en la tabla 8 y en la figura 5. En general, son coherentes con los obtenidos por Bettencourt et al. (2007), con datos de áreas funcionales de varios países, y por Escolano (2014), con las mismas variables medidas en las capitales de provincia y de autonomía en España.

De los mismos, cabe destacar que todos los valores del exponente $\beta$ son significativamente mayores que 1 (superlineares), lo que refleja que la dinámica se caracteriza por retornos crecientes, a diferencia de lo que sucede en otras actividades. Además, los ajustes son muy elevados y no hay casos extraños que se aparten claramente de la tendencia general.

Las variaciones de $\beta$ en los distintos grupos de KIS se relacionan con la intensidad de conocimiento y con los destinatarios finales de los servicios. El valor de $\beta$ es más alto en los KIS con elevada intensidad de conocimiento y en los «servicios financieros» y decrece en los «servicios orientados al mercado y a las empresas» y en «otros servicios». Los valores más bajos corresponden a las actividades de "transporte marítimo" $(\beta=0,90$; infralineal) y expresan la influencia de economías de escala; los "servicios relacionados con el empleo" y los de «bibliotecas y archivos» tienen exponentes próximos a 1 (lineal) propios de actividades relacionadas con las necesidades de las personas.

Esta relación tiene consecuencias prácticas muy importantes, pues permite conocer y predecir el tipo y el volumen de los KIS en las unidades funcionales, 
Tabla 8. Valores del exponente $\beta$ y del coeficiente de correlación $R^{2}$ del ajuste potencial entre tamaño demográfico y ocupados en los KIS en las áreas funcionales

\begin{tabular}{lccc}
\hline \multicolumn{1}{c}{ Grupo de KIS } & $\beta$ & $R^{2}$ ajustado & Actividades $\geq \beta \leq$ \\
\hline KIS con elevada intensidad tecnológica & 1,55 & 0,89 & $1,30-1,76$ \\
KIS orientados al mercado y a las empresas & 1,35 & 0,94 & $0,90-1,74$ \\
KIS: servicios financieros & 1,55 & 0,85 & $1,12-1,97$ \\
KIS: otros servicios & 1,30 & 0,89 & $1,06-1,57$ \\
\hline
\end{tabular}

Fuente: Tesorería General de la Seguridad Social.

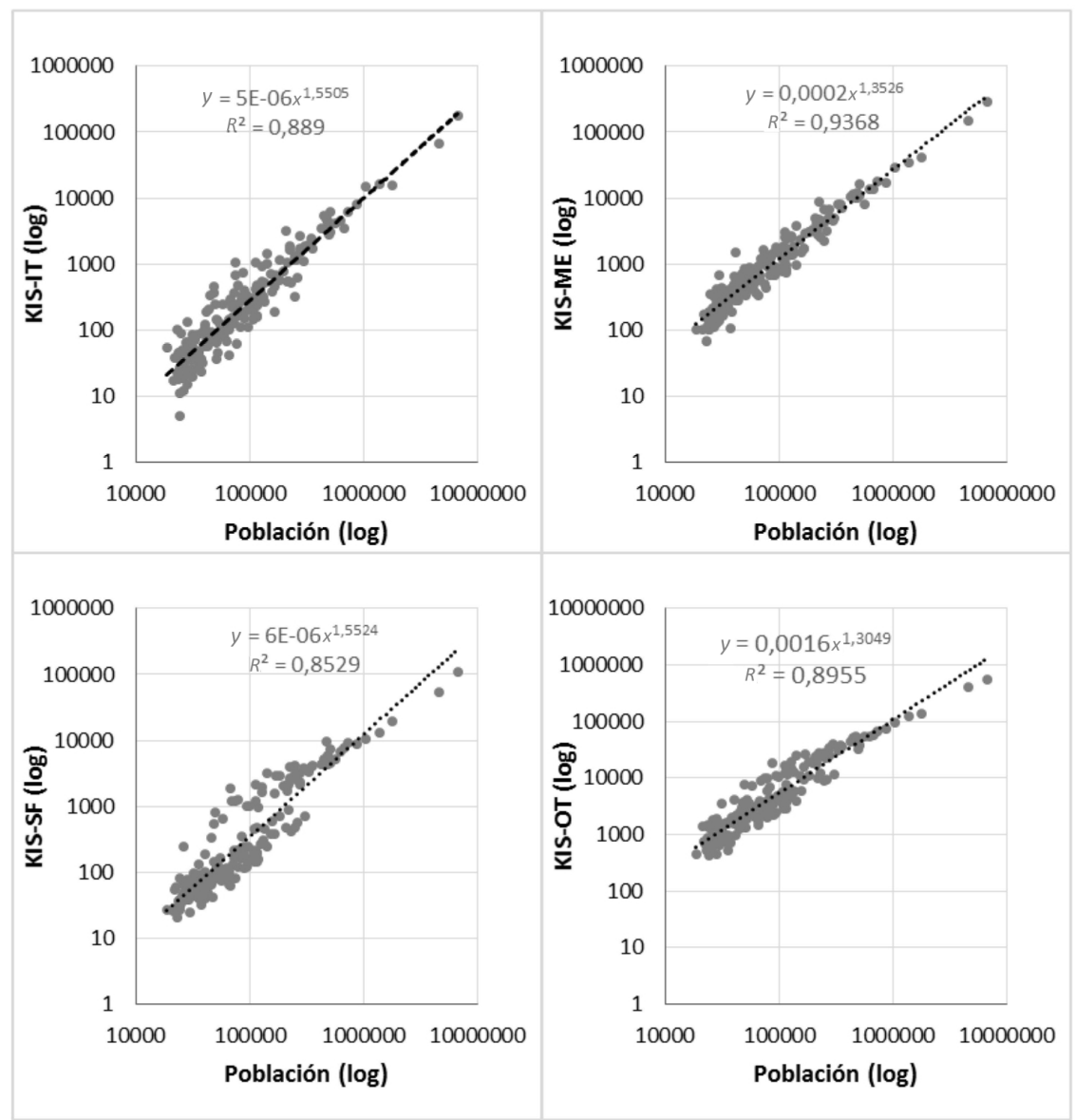

Figura 5. Diagramas de dispersión y ajuste potencial de afiliados a la Seguridad Social en los grupos de KIS y el tamaño demográfico de las áreas funcionales.

IT: elevada intensidad de conocimiento y tecnología; ME: orientados al mercado y a las empresas; SF: servicios financieros; OT: otros servicios.

Fuente: Tesorería General de la Seguridad Social e Instituto Nacional de Estadística. 
así como las condiciones socioeconómicas generales y su organización espacial, que han de reunir para que puedan desenvolverse los KIS. Por otra parte, es necesario añadir que, en España, el desarrollo de los KIS está asociado muy estrechamente al tamaño demográfico y económico de las áreas funcionales. En otras palabras: la dispersión de los KIS, especialmente los de elevada intensidad de conocimiento y tecnología, es muy limitada fuera de las grandes áreas urbanas.

\section{Consideraciones finales}

En las sociedades contemporáneas, los servicios intensivos en conocimiento (KIS) constituyen un conjunto de actividades esenciales para asegurar el bienestar de las personas, para mejorar la eficiencia de los sistemas productivos y para promover el desarrollo territorial a todas las escalas, así como la modernización de la sociedad en general.

La localización de los KIS es un factor muy relevante, tanto para la producción de estos servicios como para su prestación eficiente y equitativa. En este trabajo, queda patente un comportamiento geográfico que se caracteriza por su tendencia a conformar patrones espaciales de concentración y dispersión a varias escalas. Es un resultado coherente con los de otros estudios, lo que corrobora la validez de la metodología utilizada.

El análisis de la relación funcional entre los KIS y el tamaño demográfico de las unidades funcionales demuestra que la presencia per cápita de servicios de elevada intensidad tecnológica es más intensa en las áreas con mayor población. Este tipo de asociación indica que los servicios aquí producidos fluyen hacia el resto del territorio en que se utilizan y se consumen. Asimismo, esta concentración avala la hipótesis de que la generación de servicios relacionados con la innovación, la creatividad y el uso especializado del conocimiento requiere de un «ecosistema» socioeconómico muy diverso y complejo, de un mercado potente, de infraestructuras y equipamientos avanzados y de personal especializado, es decir, de externalidades propias de las economías de urbanización. En España, estas condiciones solo se presentan en unas pocas grandes áreas urbanas, generalmente compuestas por múltiples núcleos conectados por modernas infraestructuras de transportes y comunicaciones que conforman redes metropolitanas densas y extensas (Madrid, Barcelona, Valencia, Sevilla y Zaragoza). Dentro de las mismas, y a mayor escala, se observa que estos servicios se localizan en sectores centrales y también en zonas periféricas, en función de la orientación de cada tipo de KIS (a usuarios finales, a las empresas), de las necesidades de su producción (espacios con dotaciones técnicas adecuadas, accesibilidad, interacción con proveedores o clientes), de factores simbólicos o de prestigio y otros.

La localización de los distintos tipos de servicios intensivos en conocimiento coincide, grosso modo, con el patrón general expuesto. Es el caso de los servicios financieros y los servicios orientados a las empresas, que siguen un modelo espacial similar cuando desarrollan funciones de dirección y gestión no rutinarias. Los «otros servicios intensivos en conocimiento» y los «orientados al 
mercado» muestran configuraciones espaciales semejantes a las de la distribución de la población, cualquiera que sea la escala de análisis. La búsqueda de la proximidad al consumo (personas con perfiles específicos) y la necesidad de garantizar la provisión de algunos servicios públicos de forma eficaz y equitativa dan lugar a modelos espaciales jerárquicos gobernados, principalmente, por la distancia, la accesibilidad y la actuación de economías de escala.

Del comportamiento espacial descrito y de la relación funcional entre KIS y tamaño demográfico de las unidades funcionales, se desprende que la aparición y el desarrollo de los servicios intensivos en conocimiento, así como de innovaciones en general, precisan de determinados umbrales demográficos, económicos y de otras condiciones propias de las economías de urbanización, lo que suscita dudas respecto de la eficacia de posibles iniciativas voluntaristas enmarcadas en algunas políticas de alcance local o regional.

\section{Referencias bibliográficas}

AguilérA, Anne (2003). «La localisation des services aux entreprises dans la métropole lyonnaise: Entre centralité et diffusion». L'Espace géographique, 32, 128-140.

Albors-Garrigos, José; Hidalgo, Antonio y Hervás-Oliver, José Luis (2009). «The role of knowledge service activities (KISA) in basic agro-food processes innovation: The case of orange packers in Eastern Spain». Asian Journal of Technology Innovation [en línea], 17 (1), 31-35. <http://dx.doi.org/10.1080/19761597.2009.9668665>

Alonso, Pilar; Pueyo, Ángel; Postigo, Raúl; López, Carlos y Rubio, José Luis (2014). «La reestructuración del sector financiero». En: AlberTos, J.M. y SÁNCHEZ, J.L. Geografia de la crisis económica en España. Valencia: Universitat de València, 305-328.

Anselin, Luc (1995). "Local indicators of spatial association-LISA». Geographical Analysis, 27, 93-15.

Arbia, Giuseppe (2001). «The role of spatial effects in the empirical analysis of regional concentration». Journal of Geographical Systems [en línea], 3 (3), 271-281. $<$ http://dx.doi.org/10.1007/PL00011480>

Bade, Franz-Josef; LaAser, Claus-Friedrich y Soltwedel, Rúdiger (2004). «Urban specialization in the internet age: Empirical findings for Germany». Kiel Working Paper [en línea], 1215, 38. <http://www.econstor.eu/handle/10419/3423>.

Bailly, Antoine S. y Coffey, William J. (1994). «Localisation des services à la production et resturations économiques: Un analyse théorique». L'Espace géographique, $1,224-230$.

Bettencourt, Luis M.A.; Lobo, José; Helbing, Dirk; Kühnert, Christian y West, Geoffrey B. (2007). "Growth, innovation, scaling, and the pace of life in cities». Proceedings of the National Academy of Sciences of the United States of America [en línea], 1004 (17), 7301-7306. $<$ http://dx.doi.org/10.1073/pnas.0610172104>

Camacho, José A.; Rodríguez, Mercedes y Chica, Jorge (2012). The Regional distribution of Knowledge-Intensive Services in Europe: A spatial approach [en línea]. European Regional Science Association ERSA. ERSA Conference Papers, 21, $<$ http://www-sre.wu.ac.at/ersa/ersaconfs/ersa10/ERSA2010finalpaper277.pdf>.

Capelle-Blancard, Gunther y Tadjedine, Yamina (2009). The Location of Financial Activities: The Impact of New Tecnologies and the Financial Crisis [en línea]. París: 
Université de Paris Ouest Nanterre La Défense, Economix. Working Paper 26, 20. <http://economix.fr/pdf/dt/2009/WP_EcoX_2009-26.pdf>.

Coffey, William J. y Bailly, Antoine S. (1992). "Producer Services and Systems of Flexible Production». Urban Studies [en línea], 29 (6), 857-868. <http://dx.doi.org/10.1080/00420989220080841>

Consoli, David y Elche, Dioni (2009). Variety in the knowledge base of Business Service Sectors. Valencia: INGENIO, CSIC-UPV. Working Paper 9, 27.

Daniels, P.W. y Moulaert, F. (eds.) (1991). The changing geography of advanced producer services: Theoretical and empirical perspectives. Londres: Belhaven Press.

Davi, Maria; López-Bazo, Enrique y Barbaccia, Isidora (2009). Measurement of Agglomeration and Sapatial Effects. III World Conference of Spatial Econometrics (SEA 2009). Barcelona.

Doloreux, David; Freel, Mark y Shearmur, Richard (eds.) (2010). KnowledgeIntensive Business Services: Geography and Innovation. Surrey, UK: Ashgate. Ashgate Economic Geography Series.

Doloreux, David; Zenker, Andres y Muller, Emmanuel (2008). Services à forte intensité de conaissances, contexte régional et comportements d'innovation: Une comparaison internationale. Fraunhofer ISI. Working Papers Firms and Region: R1, 36.

Duke, Scott (s/f). Measuring agglomeration [en línea]. Harvard. Paper for Harvard Urban and Social Economics Seminar (Economics 2800b). <http://www.scottkom.com/articles/measure_agglomeration.pdf>.

Escolano, Severino (2014). «Polarización de la estructura funcional de las capitales de provincia y autonomía en España. 1981 2011». Estudios Geográficos [en línea], 75 (276), 139-175.

<http://dx.doi.org/10.3989/estgeogr.201404>

ESPON (2013). The Functional Urban Areas Database [en línea]. European Union. Technical Report, 18. <http://database.espon.eu/db2/home>.

European Commision (EC) (2007). "Towards a European strategy in support of innovation in services: Challenges and key issues for future actions». Europe INNOVA, paper n. ${ }^{\circ}$ 4. Luxemburgo: European Union, 40.

- (2012). Knowledge-intensive (business) services in Europe. Luxemburgo: European Union, 58.

Eurostat (s/f-a). Science, tecnology and innovation [en línea]. European Commision. $<$ http://ec.europa.eu/eurostat/web/science-technology-innovation/data/database>.

- (s/f-b). Statistic Explained. Glossary: Knowledge-intensive services (KIS). Eurostat.

Glaeser, Edward L. (1994). "Cities, Information, and Economic Growth». Cityscape: A Journal of Policy Development and Research, 1 (1), 9-47.

Guillain, Rachel y Le Gallo, Julie (2007). Agglomeration and dispersion of economic activities in Paris and its surroundings: An exploratory spatial data analysis [en línea]. Laboratoire d'Economie et de Gestion. CNRS. Université de Bourgogne. Document du travail. Economie: 2007-01, 36, <https://ideas.repec.org/p/lat/legeco/2007-01.html>.

Guimaraes, Paulo; Figueiredo, Octávio y Woodward, Douglas (2009). Accounting for Neighoring Effects in Measures of Spatial Concentration. Porto: Universidad de Porto. FEP Working Papers, 353, 29.

Halbert, Ludovic (2005). «Les métropoles, moteurs de la dématérialisation du système productif urbain français: Une lecture sectorielle et fonctionelle (1982-1999)». Bulletin de l'Association de Géographes Françaises [en línea], 3, 279-297. <http://dx.doi.org/10.3406/bagf.2005.2463> 
Halbert, Ludovic y Pain, Kathy (2009). «PAR-LON-doing Business in KnowledgeBased Services in Paris and Lodon: A Tale of One City?». GaWC Research Bulletin [en línea], 307. <http://www.lboro.ac.uk/gawc/publicat.html>.

Herstad, S.J. y Ebersberger, B. (20I5). «On the Link between Urban Location and the Involvement of Knowledge-Intensive Business Services Firms in Collaboration Networks». Regional Studies, 49 (7), 1160-1175.

ISAKSEN, Arne (1996). «Towards increased regional specialization?: The quantitative importance of new industrial spaces in Norway, 1970-1990». Norsk Geografisk Tidsskrift [en línea], 50 (2), 113-123.

$<$ http://dx.doi.org/10.1080/00291959608542834>

Kemprilä, Sari y MetTänen, Paula (2004). Innovation in knowledge-intensive services. 5th International CINet Conference. Sydney, 326-335.

KRÄTKE, Stefan (2007). "Metropolisation of the European Economic Territory as a consequence of Increasing Specialisation of Urban Agglomerations in the Knowledge Economy». European Planning Studies [en línea], 15 (1), 1-27. $<$ http://dx.doi.org/10.1080/09654310601016424>

Martin, Ron y Sunley, Peter (2003). «Deconstructing clusters: Chaotic concept or policy panacea?». Journal of Economic Geography [en línea], 3 (1), 5-35. <http://dx.doi.org/10.1093/jeg/3.1.5>

MÉNDEZ, Ricardo (2013). «Economía del conocimiento y nuevos contrastes territoriales en España: Una perspectiva multiescalar». Boletin de la Asociación de Geógrafos Españoles, 63, 7-32.

Méndez, Ricardo; Sánchez, Simón; Abad, Luis y García, Ignacio (2009). «Sistema urbano y sociedad del conocimiento: Hacia una tipología de ciudades españolas». Investigaciones Regionales, 16, 117-142.

Merino, Fernando y Rubalcaba, Luis (2013). «Are Knowledge-intensive Services Highly Concentrated?: Evidence from European Regions». Tijdschrift voor Economische en Sociale Geografie [en línea], 104 (2), 215-232. $<$ http://dx.doi.org/10.1111/j.1467-9663.2012.00739.x>

Miles, Ian (2003). Knowledge Intensive Services' Suppliers and Clients. Helsinky: Ministry of Trade and Industry. Report, 15, 83.

- (2008). Knowledge-Intensive Services [en línea], 23. <https://www.academia. edu/235223/Knowledge_Intensive_Services_in_Europe>.

Miller, Paul; Botham, Ron; Gibson, Hervey; Martin, Ron y Moore, Barry (2001). Business clusters in the UK: A first assessment. Report for the Department of Trade and Industry by a consortium led by Trends Business Research (3 vols.).

Moreno, Antonio y Escolano, Severino (1992). Los servicios para la producción y el consumo. Madrid: Síntesis.

Moulaert, F.; SCOTt, A.J. y FarCy, H. (1997). «Producer services and the formation of urban space». En: Cities, enterprises and society on the eve of the 21st century. (Pinter). Londres, 97-112.

O'Donoghe, Dan y Gleave, Bill (2004). "A note on methods for measuring industrial agglomeration». Regional Studies [en línea], 38 (4), 419-427. <http://dx.doi.org/10.1080/03434002000213932>

OECD (2006). Innovation and Knowledge-Intensive Service Activities. OECD.

O'Sullivan, D. y Unwin, D.J. (2010). Geographic Information Analysis. Hoboken, NJ: Wiley \& Sons, Inc. 
Pardos, E.; Gómez-Loscos, A. y Rubiera-Morollón, F. (2007). “"Do versus Buy”: Decisions in the Demand for Knowledge Intensive Business Services». The Service Industries Journal, 27 (3), 233-249.

Rodríguez, Mercedes y Camacho, José A. (2010). The role of Knowledge-intensive services in regional innovation: A European perspective. European Regional Science Association (ERSA). Conference Papers, 12.

Ruiz-Valenzuela, Jennnifer; Moreno-Serrano, Rosina y Vayá-Valcarce, Esther (2006). Concentration of the Economic Activity: Comparing Methodologies and Geographic Units [en línea]. European Regional Science Association (ERSA), 197. <https://ideas.repec.org/p/wiw/wiwrsa/ersa06p197.html>.

Salom, J.; Albertos, J.M.; Pitarch, M.D. y Delios, E. (2009). Sistema urbano e innovación industrial en el País Valenciano. Valencia: Universitat de València.

Sassen, Saskia (1991). The Global City: New York, London, Tokyo. Princeton, NJ: Princeton University Press.

SpIEzIA, Vicenzo (2003). "Measuring Regional Economies». OECD Statistics Brief, 6 (octubre), 8.

Taylor, Peter J.; Derudder, Ben; Faulconbridge, James; Hoyler, Michael y Ni, Pengfei (2014). «Advanced Producer Service Firms as Strategic Networks, Global Cities as Strategic Places». Economic Geography [en línea], 90 (3), 267-291. <http://dx.doi.org/10.1111/ecge.12040>

Tether, Bruce S. y Hipp, Christiane (2001). «Competition and Innovation Amongst Knowledge-Intensive and Other Service Firms: Evidence from Germany». En: Andersen, Brigitte; Howells, Jeremy; Hull, Richard; Miles, Ian y Roberts, Joanne. Knowledge and Innovation in the New Service Economy. Cheltenham: Edward Elgar, 49-67.

Van de Vijver, Elien; Derudder, Ben; Bassens, David y Witlox, Frank (2014). «Filling Some Black Holes: Modeling the Connection Between Urbanization, Infrastructure, and Global Service Intensity». The Professional Geographer [en línea], 66 (1), 82-90. <http://dx.doi.org/10.1080/00330124.2013.781488>

Wood, Peter (2006). «Urban Development and Knowledge-Intensive Business Services: Too Many Unanswered Questions?». Growth and Change, 37 (3), 335-361.

XIA, Haoxiang; Wang, Zhoungtuo y Dang, Yanzhong (2007). Knowledge Services: A New Research Field between KM and SSME. JAIST Press, 6. 